\title{
Social animation in complex environments ${ }^{\star}$
}

\author{
Francisco Grimaldo, Miguel Lozano, and Fernando Barber \\ Computer Science Department, University of Valencia, \\ Dr. Moliner 50, (Burjassot) Valencia, Spain \\ \{francisco.grimaldo, miguel.lozano, fernando.barber\}@uv.es
}

This work presents a market-based social model to produce good quality behavioral animations for groups of intelligent virtual agents. The social model coordinates the activities of groups of virtual characters and also includes social actions in the agent decision-making. We follow the Multi-Agent Resource Allocation approach presented in [2], where agents express their preferences using utility functions. The dynamics of social interactions is inspired by the theory of Piaget [3] over which we have implemented reciprocal task exchanges.

The social model proposed allows any agent to auction tasks in order to reallocate them so that the global social welfare can be increased. Tasks are exchanged between agents using a first-price sealed-bid (FPSB) auction model where the agents express their preferences using performance and social utility functions. The performance utility function $U_{p e r f}^{i}(\langle i \leftarrow t\rangle)$ of a bidder agent $i$ reflects the efficiency achieved when the task $t$ is allocated to the agent $i$ $(\langle i \leftarrow t\rangle)$. There can be many reasons for an agent to be more efficient: it may perform the task faster than others because of his know-how or it may be using a resource that allows several tasks to be performed simultaneously - e.g. a coffee machine in a virtual bar can be used by a waiter to make more than one coffee at the same time. Furthermore, we introduce two additional social utilities to represent the social interest in exchanging a task. We define the following social utility functions:

- Internal social utility $\left(U_{\text {int }}^{i}\left(\left\langle i \leftarrow t, j \leftarrow t_{\text {next }}\right\rangle\right)\right)$ : is the utility that a bidder agent $i$ assigns to a situation where $i$ commits to do the auctioned task $t$ so that the auctioneer agent $j$ can execute his next task $t_{\text {next }}$.

- External social utility $\left(U_{e x t}^{i}(\langle j \leftarrow t\rangle)\right)$ : is the utility that a bidder agent $i$ assigns to a situation where the auctioneer agent $j$ executes the auctioned task $t$ while $i$ continues with his current action.

Therefore, the winner determination problem has two possible candidates coming from performance (see equation 1) and sociability (see equation 2). The social winner is determined looking at the maximum social utility received to pass the task to a bidder $\left(U_{i n t}^{*}(t)\right)$ and the maximum social utility given by all bidders to the situation where the task is not exchanged but performed by the auctioneer $j\left(U_{\text {ext }}^{*}(t)\right)$. To balance task exchange, social utilities are weighted with a reciprocity matrix (see equations 3 and 4 ). We define the reciprocity

\footnotetext{
* Supported by the Spanish MEC and European Commission FEDER funds under grants TIN2006-15516-C04-04 and Consolider Ingenio-2010 CSD2006-00046.
} 
factor $w_{i j}$ for two agents $i$ and $j$, as the ratio between the number of favors i.e.tasks - that $\mathrm{j}$ has made to $\mathrm{i}$ (see equation 5 ).

$$
\begin{gathered}
\text { winner }_{\text {perf }}(t)=\left\{k \in \text { Agents } \mid U_{\text {perf }}^{i}(t)=\max _{i \in \text { Agents }}\left\{U_{\text {perf }}^{i}(\langle i \leftarrow t\rangle)\right\}\right. \\
\text { winner }_{\text {soc }}(t)= \begin{cases}j & U_{\text {ext }}^{*}(t)>=U_{\text {int }}^{*}(t) \\
i & U_{\text {ext }}^{*}(t)<U_{\text {int }}^{*}(t) \wedge U_{\text {int }}^{i}(t)=U_{\text {int }}^{*}(t)\end{cases} \\
U_{\text {int }}^{*}(t)=\max _{i \in \text { Agents }}\left\{U_{\text {int }}^{i}\left(\left\langle i \leftarrow t, j \leftarrow t_{\text {next }}\right\rangle\right) * w_{j i}\right\} \\
U_{\text {ext }}^{*}(t)=\max _{i \in \text { Agents }}\left\{U_{\text {ext }}^{i}(\langle j \leftarrow t\rangle) * w_{i j}\right\} \\
w_{i j}=\text { Favour }_{j i} / \text { Favours }_{i j}
\end{gathered}
$$

Agents choose between performance and sociability in accordance with their Sociability factor, which is the probability to select the social winner instead of the performance winner. Sociability can be adjusted in the range [0,1] to model intermediate behaviors between efficiency and total reciprocity. This can provide great flexibility when animating characters, since Sociability can be dynamically changed thus producing different behaviors depending on the world state.

The social model has been tested successfully in dynamic environments using a multi-agent simulation framework [1] that allows the definition of social BDI agents. The snapshots shown in figure 1 correspond to the simulation of a virtual bar, where groups of waiters and customers can interact and finally display complex social behaviors such as negotiation among waiters to gain access to a resource, assumption of external actions/favors, or animation of simple chats.

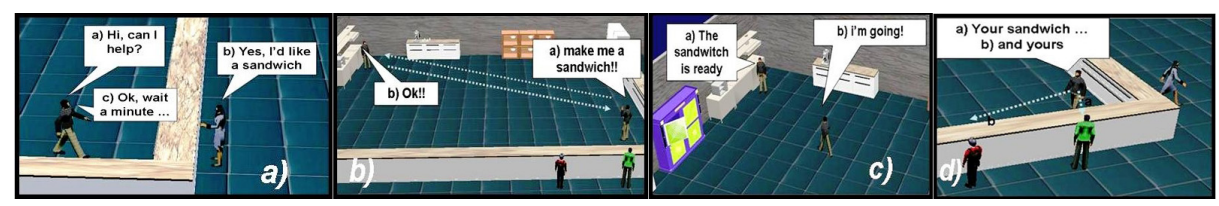

Fig. 1. Animating interaction situations.

\section{References}

1. R. H. Bordini, A. C. da Rocha, J. F. Hübner, A. F. Moreira, F. Y. Okuyama and R. Vieira. MAS-SOC: a Social Simulation Platform Based on Agent-Oriented Programming. Journal of Artificial Societies and Social Simulation, vol. 8, 2005.

2. L. M. Hogg and N. Jennings. Socially intelligent reasoning for autonomous agents. IEEE Transactions on System Man and Cybernetics, 31(5), 2001.

3. J. Piaget. Sociological studies. Routledge, London, 1995. 